\title{
THE INCREASE OF BINDING ENERGY AND ENHANCED BINDING IN NON-RELATIVISTIC QED
}

\author{
THOMAS CHEN ${ }^{1}$, VITALI VOUGALTER ${ }^{2}$, AND SEMJON A. VUGALTER $^{3}$
}

\begin{abstract}
We consider a Pauli-Fierz Hamiltonian for a particle coupled to a photon field. We discuss the effects of the increase of the binding energy and enhanced binding through coupling to a photon field, and prove that both effects are the results of the existence of the ground state of the self-energy operator with total momentum $P=0$.
\end{abstract}

\section{INTRODUCTION.}

We consider a charged particle coupled to a photon field that interacts with an external potential in nonrelativistic QED. This system will be described by a Pauli-Fierz Hamiltonian, whereas neglecting the radiation effects, one obtains a corresponding Schrödinger operator. In the present paper, we discuss two closely related questions:

(1) Does the interaction with a quantized radiation field increase binding abilities of a potential (whether the Pauli-Fierz Hamiltonian can have a ground state if the Schrödinger operator with the same potential does not)?

(2) If the corresponding Schrödinger operator has discrete spectrum, should the binding energy (the difference between the infimum of the energy with and without potential, measured in units $m c^{2}$, where $m$ is the bare electron mass) increase if the interaction with the radiation field is considered?

We emphasize here that the asserted increase of binding energy holds with respect to the bare electron mass. In physical experiments, the binding energy is usually measured in units $m_{\text {phys }} c^{2}$, where $m_{\text {phys }}$ is the rest mass of the free infraparticle (comprising the free electron together with a cloud of low-energetic photons that it binds). In these units, the binding energy decreases.

\footnotetext{
${ }^{1}$ Courant Institute of Mathematical Sciences, New York University. Supported by a Courant Instructorship.

${ }^{2}$ Department of Mathematics and Statistics, McMaster University.

3 Mathematisches Institut, LMU München.
} 
Physical intuition suggests that the answer to both (1) and (2) should be in the affirmative. The free infraparticle binds a larger quantity of low-energetic photons than the confined particle, and thus possesses a larger effective mass. In order for the particle to leave the potential well, an additional energetic effort, proportional to the difference of the two effective masses, is therefore necessitated, relative to the situation without coupling to the quantized electromagnetic field.

Recently, problems 1 and 2 were actively studied in the mathematical literature. First, let us mention the paper by M. Griesemer, E. Lieb, M. Loss (GLL), where the authors proved that the binding energy cannot be decreased by the photon field. Another (and more important) achievement of GLL is a criterion for a Pauli-Fierz Hamiltonian to have a ground state. This criterion will be also used in the present paper. The investigation of enhanced binding (Problem 1) was started by F. Hiroshima and H. Spohn ( [HS1]), who considered the Pauli-Fierz Hamiltonian in the dipole approximation. In this approximation, the dependence of the magnetic vector potential on the coordinates of the particle is neglected. They proved the existence of enhanced binding for sufficiently large values of the coupling parameter $\alpha$ (which is the fine structure constant, $\alpha \approx \frac{1}{137}$ in nature) .

A different approach was implemented in [HVV]. The Pauli-Fierz operator without spin term $(\sqrt{\alpha} \sigma \cdot B)$ was studied with a potential, for which the corresponding Schrödinger operator does not have discrete eigenvalues, but which is very close to the appearance of the first eigenvalue. The first step was to estimate the self-energy for small $\alpha$ with an error of the order $o\left(\alpha^{2}\right)$, and then it was proved that by adding the potential, one gets a shift $C \alpha^{2}$ of the infimum of the spectrum, which for small $\alpha$ implies the existence of the ground state GLL. This approach was further developed in a recent preprint $[\mathrm{CH}]$, where the case of a particle with spin was considered.

The increase of the binding energy for the Coulomb potential $e|x|^{-1}$ in a model situation, where the electron charge $e$ is constant, but $\alpha$ tends to zero, was proved by C.Hainzl $[\mathrm{H}$, by controlling the expansion of the ground state energy to order $o\left(\alpha^{2}\right)$. To establish the corresponding result for the physical case $e=-\sqrt{\alpha}$ by the methods of $[\mathrm{H}]$, one would have to control the expansion at least up to order $O\left(\alpha^{3}\right)$. On the other hand, simple physical arguments show that this effect is caused by the form of the self-energy operator and does not depend on the coefficient of the potential. For fixed $\alpha$, the increase of the binding energy should exist for all values of $e<0$. 
It is nessesary to emphasize that the methods of [HVV] and $[\mathrm{H}]$ are asymptotic in $\alpha$, and that they can hardly be generalized in a manner to cover the physical case, where $\alpha$ is a fixed constant. Studying this case requires a different strategy, which is not based on asymptotic expansions in $\alpha$. The work at hand is the first attempt to develop such methods. We prove two very simple theorems, showing that both effects take place if the self-energy operator, restricted to the states with total momentum $P=0$ (operator $T_{0}$ ), has a ground state. The proof of these two statements is based on direct variational estimates of the binding energy, and the results are independent of $\alpha$. Establishing the connection between the existence of the ground state of the operator $T_{0}$, and the existence of enhanced and increased binding is the main achievement of the present paper.

The existence of the ground state of $T_{0}$ is a problem important for different aspects of nonrelativistic QED, and has been solved in $\mathrm{C}$ for small $\alpha$. Applying the results of $[\mathrm{C}$ and some generalizations thereof stated in the Appendix of the present paper, we immediately obtain a new, very simple proof of enhanced binding for small $\alpha$, in both the spin and spinless cases, as well as the proof of the increase of the binding energy for all potentials, for which the corresponding Schrödinger operator has a ground state. In particular, we prove the increase of binding energy for the Coulomb potential $\left(e|x|^{-1}\right)$, for all $e<0$.

\section{Definitions AND MAIN THEOREMS.}

The Hamiltonian for an electron interacting with the quantized radiation field and a given external potential $V(x), x \in \mathbb{R}^{3}$ is

$$
H=T+V(x)
$$

where

$$
T=(p+\sqrt{\alpha} A(x))^{2}+g \sqrt{\alpha} \sigma \cdot B(x)+H_{f} .
$$

We fix units such that $\hbar=c=1$ and the electron mass $m=\frac{1}{2}$, $\alpha=e^{2}$ is the "fine structure" constant, $e$ is the electron charge. The natural value of $\alpha$ is $\simeq \frac{1}{137}$, however, as usual [BFS, HS1] we will think about $\alpha$ as a parameter in the operator $T$. The main results of this paper (Theorems 1,2) are true for all $\alpha>0$. An artificial parameter $g$, which can attain the values of either 0 or 1 is introduced to describe both the spin $(g=1)$ and the spinless $(g=0)$ cases. As usual $\sigma=\left(\sigma_{1}, \sigma_{2}, \sigma_{3}\right)$ is the vector of Pauli matrices, $p=-i \nabla_{x}$, $B(x)=\operatorname{curl} A(x)$. The magnetic vector potential $A(x)$ is given by

$$
A(x)=\sum_{\lambda=1,2} \int_{\mathbb{R}^{3}} \frac{\chi(|k|)}{2 \pi|k|^{1 / 2}} \varepsilon_{\lambda}\left[a_{\lambda}(k) e^{i k x}+a_{\lambda}^{*}(k) e^{-i k x}\right] d k,
$$


where the operators $a_{\lambda}, a_{\lambda}^{*}$ satisfy the usual commutation relations

$$
\left[a_{\nu}(k), a_{\lambda}^{*}(q)\right]=\delta(k-q) \delta_{\lambda, \nu}, \quad\left[a_{\lambda}(k), a_{\nu}(q)\right]=0 .
$$

The vectors $\varepsilon_{\lambda}(k) \in \mathbb{R}^{3}$ are the two possible orthonormal polarization vectors perpendicular to $k$.

The function $\chi(|k|)$ in (2.3) describes the ultraviolet cutoff on the wavenumbers $|k|$. The only restriction on $\chi(|k|)$, which we need at the moment, is $\chi(|k|) \equiv 0$ for $|k|>\Lambda$ with some $\Lambda>0$.

The photon field energy $H_{f}$ is given by

$$
H_{f}=\sum_{\lambda=1,2} \int_{\mathbb{R}^{3}}|k| a_{\lambda}^{*}(k) a_{\lambda}(k) d k .
$$

Regarding the potential $V(x)$ we assume that $V(x)=V(|x|), V(x) \in$ $\mathcal{L}_{2, l o c}\left(\mathbb{R}^{3}\right),|V(x)| \leq C$ for $|x| \geq a$ with some constants $C>0, a>0$ and $|V(x)| \rightarrow 0$ as $|x| \rightarrow \infty$. For $g=1$ the operators $T$ and $H$ are considered on the space

$$
\mathcal{H}=\mathcal{L}_{2}\left(\mathbb{R}^{3} ; \mathbb{C}^{2}\right) \otimes \mathcal{F},
$$

where $\mathcal{F}$ is the Fock space for the photon field.

If $g=0$ the corresponding space is

$$
\mathcal{H}=\mathcal{L}_{2}\left(\mathbb{R}^{3}\right) \otimes \mathcal{F}
$$

According to Hi the operator $H$ is semibounded from below and essentially selfadjoint.

For an arbitrary selfadjoint operator $\mathcal{A}$ let $s(\mathcal{A})$ and $s_{\text {disc }}(\mathcal{A})$ be the spectrum and the discrete spectrum of $\mathcal{A}$ and let

$$
E_{0}=\inf s(T), \quad E_{1}=\inf s(H) \quad \text { and } \quad \Delta_{E}=E_{0}-E_{1} .
$$

To compare the binding energy in the presence of the photon field $\left(\Delta_{E}\right)$ and the binding energy without photon field let us consider the Schrödinger operator

$$
h=-\Delta+V(|x|)
$$

with the same potential $V(|x|)$ as in (2.1). Denote by $-e_{0}$ the lowest eigenvalue of the operator $h$ (if $s_{d i s c}(h)=\emptyset, e_{0}=0$ ). Obviously $e_{0}$ is the binding energy for the Schrödinger operator. According to GLL]

$$
\Delta_{E} \geq e_{0} .
$$

In the present paper it will be proved, that under some conditions, the strong inequality

$$
\Delta_{E}>e_{0}
$$

holds .

Suppose now that the potential $V(|x|)$ is short-range, $|V(|x|)| \leq$ $c(1+|x|)^{-2-\delta}, \delta>0$ and its negative part is nontrivial. We can write 
$V(|x|)$ as $\beta V_{0}(|x|)$, where $V_{0}(|x|)$ is a short-range potential, which we will keep fixed and $\beta>0$ is a coupling constant. Denote by $\beta_{0}$ the minimal value of the coupling constant $\beta$, such that for $\beta>\beta_{0}$, the operator $h$ with the potential $\beta V_{0}(|x|)$ has nonempty discrete spectrum and by $\beta_{1}$ the minimal value of the coupling constant such that for all $\beta>\beta_{1}$ operator $H$ with the same potential has a ground state.

It will be proved that under the same conditions as we need for (2.6)

$$
\beta_{1}<\beta_{0} .
$$

This means that the photon field increases the binding abilities of potentials. To formulate those conditions, we need to introduce some more definitions.

First, notice that the operator $T$ is translationally invariant. It commutes with the operator of the total momentum

$$
P_{t o t}=p_{e l} \otimes I_{f}+I_{e l} \otimes P_{f},
$$

where $p_{e l}$ and $P_{f}=\sum_{\lambda=1,2} \int d^{3} k k a_{\lambda}^{*}(k) a_{\lambda}(k)$ denote the electron and the photon momentum operators respectively.

The Hilbert space $\mathcal{H}$ can be written as a direct integral

$$
\mathcal{H}=\int^{\oplus} d^{3} P \mathcal{H}_{P},
$$

where $\mathcal{H}_{P}$ are the fibre Hilbert spaces associated to the fixed values $P$ of the conserved momentum, which are invariant under space and time translations. For any fixed value $P$ of the total momentum the restriction of $T$ to the fibre space $\mathcal{H}_{P}$ is given by the operator

$$
T_{P}=\left(P-P_{f}+\sqrt{\alpha} A(0)\right)^{2}+\sqrt{\alpha} g \sigma \cdot B(0)+H_{f} .
$$

The operator $T_{P}$ with $P=0$ (we will call it $T_{0}$ ) plays an especially important role.

The main theorems will be proved under the following assumption.

Condition 1. There is an element $\psi_{0} \in \mathcal{H}_{0}$, satisfying

$$
T_{0} \psi_{0}=E_{0} \psi_{0} .
$$

We note that Condition 1 contains two parts. For it to be satisfied, we must first have

$$
E_{0}=\inf s(T)=\inf s\left(T_{0}\right),
$$

and secondly, $E_{0}$ has to be in the point spectrum of the operator $T_{0}$. These issues will be further addressed after the statements of the theorems. 
Theorem 1. (The increase of the binding energy) Let Condition 1 be satisfied and $s_{\text {disc }}(h) \neq \emptyset$. Then

$$
\Delta_{E}>e_{0} .
$$

Theorem 2. (Enhanced binding) Let Condition 1 be fulfilled, and let $V(|x|)=\beta V_{0}(|x|)$ be a short-range potential with the properties described above. Then

$$
\beta_{1}<\beta_{0} .
$$

Remark. 1. Let us first discuss theorems 1 and 2 for the spinless case $g=0$. The fact that $\inf s\left(T_{0}\right)=E_{0}$ was proved by J. Fröhlich for all $\alpha$. Recently, it was proved in $[\mathrm{C}]$ that for small $\alpha$, and an ultraviolet cutoff $\chi \in C^{1}\left(\mathbb{R}_{+}\right), E_{0}$ is contained in the point spectrum of $T_{0}$. Thus, under these assumptions, the conditions of theorems 1 and 2 are clearly fulfilled.

2. If $g=1$ (inclusion of particle spin), both parts of condition 1 remain true. This follows from the generalizations of $[\mathrm{C}]$ outlined in the Appendix of the present work.

3. It may be useful to briefly comment on the case for models that include an infrared regularization. It follows from $[\mathrm{C}]$ that Condition 1 is satisfied by models that are infrared regularized by an infrared cutoff function in $A(0)$ that vanishes nowhere on $(0, \Lambda)$ (this technical requirement is used for the Ward-Takahashi identities within the operator-theoretic renormalization group scheme of $[\mathrm{C}]$ ). Consequently, theorems 1 and 2 hold for these models. If the infrared cutoff vanishes in an open neighborhood of $\{0\}$, or if it is incorporated by adding a small photon mass, the methods of $[\mathrm{F}]$ can be applied to verify Condition 1.

Corollary 1. Let the ultraviolet cutoff $\chi(|k|)$ be fixed and have bounded first derivatives. Then one can find a number $\alpha_{0}$ independent of the potential $V(|x|)$, such that for all $0<\alpha<\alpha_{0}$ the following two statements hold:

i) if $e_{0} \neq 0$ then $\Delta_{E}>e_{0}$;

ii) if $V(|x|)=\beta V_{0}(|x|)$ is a short-range potential satisfying the same condition as formulated above, then $\beta_{1}<\beta_{0}$.

\section{Proof of Theorem 1.}

To prove the theorem, we shall construct a trial function $\varphi \in \mathcal{H}$, such that

$$
(H \varphi, \varphi)<\left(E_{0}-e_{0}\right)\|\varphi\|^{2} .
$$


Let $\psi_{0}$ be the ground state of the operator $T_{0}$

$$
T_{0} \psi_{0}=E_{0} \psi_{0}, \quad\left\|\psi_{0}\right\|_{\mathcal{H}_{0}}=1 .
$$

We will need the following fact.

Lemma 1. Assume $\psi_{0}$ and $E_{0}$ as in Condition 1. Then, the relation

$$
\left\|\left(P_{f}-\sqrt{\alpha} A(0)\right) \psi_{0}\right\| \neq 0
$$

holds ${ }^{1}$.

Proof. By contradiction. Assume that (3.3) is incorrect. Then,

$$
P_{f} \psi_{0}=\sqrt{\alpha} A(0) \psi_{0},
$$

and the magnetic term yields

$$
\begin{aligned}
\sigma \cdot B_{f}(0) \psi_{0} & =i \sigma\left(P_{f} \wedge A(0)+A(0) \wedge P_{f}\right) \psi_{0} \\
& =i \sigma\left(\frac{1}{\sqrt{\alpha}} P_{f} \wedge P_{f}+\sqrt{\alpha} A(0) \wedge A(0)\right) \psi_{0} \\
& =0 .
\end{aligned}
$$

Thus,

$$
T_{0} \psi_{0}=H_{f} \psi_{0}=E_{0} \psi_{0} .
$$

But this yields a contradiction, since the only eigenvector of $H_{f}$ is the Fock vacuum, which fails to satisfy (3.4) if $\alpha \neq 0$. This establishes the Lemma.

Thus,

$$
\left(P_{f}-\sqrt{\alpha} A(0)\right) \psi_{0}=\sum_{i=1}^{3} e_{i} \psi_{0 i} \not \equiv 0,
$$

where $e_{i}$ is the orthonormal basis in $\mathbb{R}^{3}$. Without loss of generality, we assume $\psi_{01} \not \equiv 0$. Let $\tilde{\psi}_{01}$ be a fixed function in $D\left(T_{0}\right)$, such that $\left\|\tilde{\psi}_{01}\right\|_{\mathcal{H}_{0}}=1$ and

$$
\Re\left(\tilde{\psi}_{01}, \psi_{01}\right)_{\mathcal{H}_{0}} \geq \frac{1}{2}\left\|\psi_{01}\right\|_{\mathcal{H}_{0}} .
$$

For our estimates it is more convenient to consider the functions $\psi_{0}$ and $\tilde{\psi}_{01}$ in the coordinate (for photons) representation. Let $\xi_{i} \in \mathbb{R}^{3}$ be the position vectors of photons. A function $\psi \in \mathcal{H}_{0}$ can be written as

$$
\psi=\underset{n}{\oplus} \psi_{n}\left(s, \xi_{1}, \ldots, \xi_{n}, \lambda_{1}, \ldots, \lambda_{n}\right),
$$

\footnotetext{
${ }^{1}$ Notice that $\left(P_{f}-\sqrt{\alpha} A(0)\right) \psi_{0}$ is a 3 -component vector with components $\psi_{0 i} \in \mathcal{H}_{0} i=1,2,3$. As usual $\left\|\left(P_{f}-\sqrt{\alpha} A(0)\right) \psi_{0}\right\|=\left(\sum_{i=1}^{3}\left\|\psi_{0 i}\right\|_{\mathcal{H}_{0}}^{2}\right)^{1 / 2}$.
} 
where $s$ is the electron spin and $\lambda_{i}$ are the polarization vectors of photons.

For $x \in \mathbb{R}^{3}$ and an arbitrary element $\psi \in \mathcal{H}_{0}$ we define the operator $S_{x}$ and the function $\psi_{x} \in \mathcal{H}_{0}$ by the formula

$$
\psi_{x}=S_{x} \psi=\bigoplus_{n} \psi_{n}\left(s, \xi_{1}-x, \ldots, \xi_{n}-x, \lambda_{1}, \ldots, \lambda_{n}\right) .
$$

Denote by $f_{0}(|x|)$ the real normalized eigenfunction of the operator $h$ corresponding to the lowest eigenvalue and let $f_{1}(x) \in C_{0}^{2}\left(\mathbb{R}^{3}\right)$ be the function with the following properties:

i) $f_{1}(x)$ is real,

ii) $\left\|f_{1}(x)\right\|=1$

iii) $f_{1}(x)$ is symmetric with respect to the reflections $x_{2} \leftrightarrow-x_{2}$ and $x_{3} \leftrightarrow-x_{3}$ and antisymmetric with respect to the reflection $x_{1} \leftrightarrow-x_{1},^{2}$

iv)

$$
\left(\frac{\partial f_{0}(|x|)}{\partial x_{1}}, f_{1}(x)\right)>\frac{1}{2}\left\|\frac{\partial f_{0}}{\partial x_{1}}\right\| .
$$

Now we are ready to define the trial function $\varphi$. Let $\eta$ be a real valued parameter, which will be specified later and let

$$
\varphi=f_{0}(x) \psi_{x 0}+i \eta f_{1}(x) \tilde{\psi}_{x 01}
$$

where the functions $f_{0}, f_{1}, \psi_{0}, \tilde{\psi}_{01}$ are defined above, $\psi_{x 0}=S_{x} \psi_{0}$ and $\tilde{\psi}_{x 01}=S_{x} \tilde{\psi}_{01}$. Our next goal is to prove (3.1) for $\eta$ small and negative.

Obviously

$$
\begin{aligned}
(H \varphi, \varphi) & =\left(H f_{0}(x) \psi_{x 0}, f_{0}(x) \psi_{x 0}\right)-\eta^{2}\left(H f_{1}(x) \tilde{\psi}_{x 01}, f_{1}(x) \tilde{\psi}_{x 01}\right) \\
& -2 \eta \Im\left(H f_{0}(x) \psi_{x 0}, f_{1}(x) \tilde{\psi}_{x 01}\right)
\end{aligned}
$$

The second term in the right side of (3.10) can be estimated from above as $c_{0} \eta^{2}$ with a constant $c_{0}$ independent of $\eta$. Let us evaluate the first term. Notice that

$$
p f_{0}(x) \psi_{x 0}=\left(p f_{0}(x)\right) \psi_{x 0}-f_{0}(x) P_{f} \psi_{x 0}
$$

which implies

$$
\begin{aligned}
\left(H f_{0}(x) \psi_{x 0}, f_{0}(x) \psi_{x 0}\right) & =\left\|\left.p f_{0}(x)\right|^{2}\right\| \psi_{x 0} \|_{\mathcal{H}_{0}}^{2} \\
+\left(f_{0}(x) V(|x|), f_{0}(x)\right)\left\|\psi_{x 0}\right\|_{\mathcal{H}_{0}}^{2} & +\left\|f_{0}(x)\right\|^{2}\left(T \psi_{0}, \psi_{0}\right)_{\mathcal{H}_{0}} \\
& =\left(E_{0}-e_{0}\right)\left\|f_{0}(x) \psi_{0}\right\|^{2}
\end{aligned}
$$

\footnotetext{
${ }^{2}$ Everywhere in the paper $x=\left(x_{1}, x_{2}, x_{3}\right) \in \mathbb{R}^{3}$.
} 
Here we also used the orthogonality

$$
\left(\frac{\partial f_{0}}{\partial x_{i}}, f_{0}\right)=0 \quad i=1,2,3 .
$$

To estimate the last term in (3.10) recall that because of the symmetry $\left(f_{0}, f_{1}\right)=0, \quad\left(V(|x|) f_{0}, f_{1}\right)=0,\left(\Delta f_{0}, f_{1}\right)=0, \quad\left(\frac{\partial f_{0}}{\partial x_{i}}, f_{1}\right)=0 \quad i=2,3$.

Hence

$$
\begin{aligned}
-2 \eta \Im\left(H f_{0}(x) \psi_{x 0}, f_{1}(x) \tilde{\psi}_{x 01}\right) & =2 \eta\left(\frac{\partial f_{0}}{\partial x_{1}}, f_{1}\right) \Re\left(\psi_{01}, \tilde{\psi}_{01}\right) \\
& \leq \frac{\eta}{2}\left\|\psi_{01}\right\|_{\mathcal{H}_{0}}\left\|\frac{\partial f_{0}}{\partial x_{1}}\right\|^{2}
\end{aligned}
$$

for $\eta<0$.

Combining (3.12), (3.7) and (3.10) we arrive at

$$
(H \varphi, \varphi) \leq\left(E_{0}-e_{0}\right)\left\|f_{0}\right\|^{2}+c_{0} \eta^{2}+\frac{\eta}{2}\left\|\frac{\partial f_{0}}{\partial x_{1}}\right\|^{2}\left\|\psi_{01}\right\|_{\mathcal{H}_{0}} .
$$

To complete the proof of Theorem 1 notice that

$$
\|\varphi\|_{\mathcal{H}}^{2}=\left\|f_{0}(x)\right\|^{2}+\eta^{2}\left\|f_{1}(x)\right\|^{2}=\left\|f_{0}(x)\right\|^{2}+\eta^{2}
$$

and hence

$$
(H \varphi, \varphi) \leq\left(E_{0}-e_{0}\right)\|\varphi\|_{\mathcal{H}}^{2}+\eta^{2}\left[\left|E_{0}-e_{0}\right|+c_{0}\right]+\frac{\eta}{6}\left\|\nabla f_{0}\right\|^{2}\left\|\psi_{01}\right\|_{\mathcal{H}_{0}}
$$

which for

$$
0>\eta>-\frac{1}{6}\left\|\nabla f_{0}(x)\right\|^{2}\left\|\psi_{01}\right\|_{\mathcal{H}_{0}}\left[\left|E_{0}-e_{0}\right|+c_{0}\right]^{-1}
$$

yields (3.1).

\section{Proof of Theorem 2 .}

We shall prove that for $\beta=\beta_{0}$

$$
\inf s(H)<E_{0} \text {. }
$$

The statement of the theorem follows from (4.1) and the variational principle.

Let $0 \leq \gamma<1, V_{0}(|x|)$ satisfies the conditions of the theorem,

$$
h_{\gamma, \beta_{0}}=-(1-\gamma) \Delta+\beta_{0} V_{0}(|x|) .
$$

The operator $h_{\gamma, \beta_{0}}$ does not have discrete eigenvalues for $\gamma=0$, but for all $0<\gamma<1$ it has at least one real spherically symmetric eigenfunction $f_{\gamma}(|x|)$. 
Let $\varphi$ be the function defined by (3.8) with $f_{0}(|x|)$ replaced by $f_{\gamma}(|x|)$ and $f_{1}(|x|)$ replaced by $f_{\gamma, 1}(x)=\frac{\partial}{\partial x_{1}} f_{\gamma}(|x|)$. We shall prove that for $\gamma>0,|\eta|$ sufficiently small $(\eta<0)$, the inequality

$$
(H \varphi, \varphi)<E_{0}\|\varphi\|^{2}
$$

is true.

To this end, first let us recall some properties of the functions $f_{\gamma}$ for small $\gamma$ (see, for example, $[\mathrm{VZ}]$ ).

Let $\mathbb{B}$ be the closure of the space $C_{0}^{\infty}\left(\mathbb{R}^{3}\right)$ in the norm $\|\psi\|_{\mathbb{B}}=\|\nabla \psi\|$. The equation

$$
-\Delta \psi+\beta_{0} V_{0}(x) \psi=0
$$

has a unique spherically symmetric solution $\bar{\psi} \in \mathbb{B},\|\bar{\psi}\|_{\mathbb{B}}=1$. This solution (which is called the virtual level or zero-resonance ) satisfies : $\Delta \bar{\psi}, V_{0}(|x|) \bar{\psi} \in \mathcal{L}^{2}\left(\mathbb{R}^{3}\right)$. Assume that the eigenfunctions $f_{\gamma}(x)$ are normalized by the condition $\left\|\nabla f_{\gamma}(x)\right\|=1$. Then $[\mathrm{VZ}$ ]

$$
\left\|\Delta f_{\gamma}-\Delta \bar{\psi}\right\| \rightarrow 0 \text { as } \quad \gamma \rightarrow 0,
$$

which implies the inequality

$$
\left\|\Delta f_{\gamma}\right\| \leq 2\|\Delta \bar{\psi}\|\left\|\nabla f_{\gamma}\right\|=C_{0}\left\|\nabla f_{\gamma}\right\|
$$

for all $\gamma$ small and $C_{0}$ independent of $\gamma$. Let us turn directly to estimating the quadratic form $(H \varphi, \varphi)$.

Similarly to (3.10) we have

$$
\begin{aligned}
(H \varphi, \varphi) & =\left(H f_{\gamma}(x) \psi_{x 0}, f_{\gamma}(x) \psi_{x 0}\right)-\eta^{2}\left(H f_{\gamma, 1}(x) \tilde{\psi}_{x 01}, f_{\gamma, 1}(x) \tilde{\psi}_{x 01}\right) \\
& -2 \eta \Im\left(H f_{\gamma}(x) \psi_{x 0}, f_{\gamma, 1}(x) \tilde{\psi}_{x 01}\right) .
\end{aligned}
$$

For the second term in the right side of (4.5) the bound holds :

$$
\begin{aligned}
\left|\eta^{2}\left(H f_{\gamma, 1}(x) \tilde{\psi}_{x 01}, f_{\gamma, 1}(x) \tilde{\psi}_{x 01}\right)\right| & \leq \eta^{2}\left\{C_{1}\left\|f_{\gamma, 1}\right\|^{2}+C_{2}\left\|\nabla f_{\gamma, 1}\right\|^{2}\right\} \\
& \leq \eta^{2}\left[C_{1}+2 C_{2} C_{0}\right]\left\|\nabla f_{\gamma}\right\|^{2}
\end{aligned}
$$

where the constants $C_{1}$ and $C_{2}$ depend on $\tilde{\psi}_{01}$, but do not depend on $f_{\gamma}$, and $C_{0}$ is the constant from (4.4).

Analogously to (3.12) and (4.2) respectively

$$
\left(H f_{\gamma}(x) \psi_{x 0}, f_{\gamma}(x) \psi_{x 0}\right)=E_{0}\left\|f_{\gamma}(x)\right\|^{2}+\left(h_{0, \beta_{0}} f_{\gamma}(x), f_{\gamma}(x)\right)
$$

and

$$
\begin{aligned}
-2 \eta \Im\left(H f_{\gamma}(x) \psi_{x 0}, f_{\gamma, 1}(x) \tilde{\psi}_{x 01}\right) & \leq-\frac{|\eta|}{2}\left\|\frac{\partial f_{\gamma}}{\partial x_{1}}\right\|^{2}\left\|\psi_{01}\right\|_{\mathcal{H}_{0}} \\
& =-\frac{|\eta|}{6}\left\|\psi_{01}\right\|_{\mathcal{H}_{0}}\left\|\nabla f_{\gamma}\right\|^{2},
\end{aligned}
$$

recalling that $\left\|\psi_{0}\right\|_{\mathcal{H}_{0}}=1$. 
Combining (4.5) with (4.6)-(4.8) we obtain

$$
\begin{aligned}
(H \varphi, \varphi) & \leq E_{0}\left\|f_{\gamma}(x)\right\|^{2}+\left(h_{0, \beta_{0}} f_{\gamma}(x), f_{\gamma}(x)\right) \\
& +\left\{\eta^{2}\left[C_{1}+2 C_{2} C_{0}\right]-\frac{|\eta|}{6}\left\|\psi_{01}\right\|_{\mathcal{H}_{0}}\right\}\left\|\nabla f_{\gamma}(x)\right\|^{2} \\
& \leq E_{0}\|\varphi\|^{2}+(1-\delta)\left\|\nabla f_{\gamma}\right\|^{2}+\beta_{0}\left(V_{0} f_{\gamma}(x), f_{\gamma}(x)\right),
\end{aligned}
$$

where

$$
\delta=\frac{|\eta|}{6}\left\|\psi_{01}\right\|_{\mathcal{H}_{0}}-\eta^{2}\left[\frac{1}{3}\left|E_{0}\right|+C_{1}+2 C_{2} C_{0}\right] .
$$

Here we used the relation

$$
\|\varphi\|_{\mathcal{H}}^{2}=\left\|f_{\gamma}(x)\right\|^{2}+\eta^{2}\left\|f_{\gamma, 1}\right\|^{2}\left\|\tilde{\psi}_{01}\right\|_{\mathcal{H}_{0}}^{2}=\left\|f_{\gamma}(x)\right\|^{2}+\frac{1}{3} \eta^{2}\left\|\nabla f_{\gamma}\right\|^{2} .
$$

Notice, that for $|\eta|$ small $\delta>0$, and to complete the proof of the theorem it suffices to take $0<\gamma \leq \delta$.

\section{ApPEndiX}

In this appendix, we comment on the following statements that were central for the applications of theorems 1 and 2 in the case $g=1$. For $\alpha$ sufficiently small:

(i) The global minimum of $\inf \operatorname{spec}\left\{T_{P}\right\}$ for $P \in \mathbb{R}^{3}$ is attained at $P=0$.

(ii) There exists a ground state $\psi_{0} \in \mathcal{H}_{0}$ of $T_{0}$ for $g=1$.

Let us comment on the proof. We recall that

$$
T_{P}=\left(P-P_{f}-\sqrt{\alpha} A\right)^{2}+i g \sqrt{\alpha} \sigma \cdot\left(P_{f} \wedge A+A \wedge P_{f}\right)+H_{f},
$$

where $A \equiv A(0)$. Let us to begin with include an artificial infrared regularization in the quantized electromagnetic vector potential, which acts like a momentum cutoff at small $\rho>0$ (some requirements on its precise form are formulated in [C]), by which we substitute $A \rightarrow A(\rho)$ (under a slight abuse of notation), and $T_{P} \rightarrow T_{P}(\rho)$. Then, in addition to (i) and (ii), we claim that

(iii) For all $\rho>0$, and $|P| \geq 0$ and $\alpha$ sufficiently small,

$$
E(P, \rho):=\inf \operatorname{spec}\left\{T_{P}(\rho)\right\}
$$

is an eigenvalue, whose eigenspace in $\mathcal{H}_{P} \cong \mathbb{C}^{2} \otimes \mathcal{F}$ has dimension 2. Assume $\psi_{P}(\rho) \in \mathbb{C}^{2} \otimes \mathcal{F}$ is an eigenvector. Then, if $P=0, \psi_{0}(\rho)$ tends to a ground state $\psi_{0}(0) \in \mathbb{C}^{2} \otimes \mathcal{F}$ in the limit $\rho \rightarrow 0$. We note that the last statement is false in the case $|P|>0$; if $|P|>0, T_{P}(0)$ fails to have a ground state in $\mathbb{C}^{2} \otimes \mathcal{F}$. 
(iv) For some $\delta>0,0<P_{c}<1$ and $\alpha$ sufficiently small,

$$
\left|\partial_{P}^{b}\left(E(P, \rho)-P^{2}\right)\right| \leq C \alpha^{\delta}
$$

uniformly for $\rho \geq 0$, with $b=0,1,2$, and all $P,|P|<P_{c}$.

The detailed proof of these results will be published separately. The degeneracy of the ground state energy has recently been proved by F. Hiroshima and H. Spohn, [HS2], for the case where the photons have a small mass. We will here briefly sketch the strategy, which is an extension of [C]. It uses the operator-theoretic renormalization group based on the smooth Feshbach map, [BCFS1, BCFS2, C], and its framework can be roughly outlined as follows. One introduces a certain Banach space $\mathcal{W}$ of bounded operators acting on the Hilbert space $\mathbf{1}_{2} \otimes$ $\chi\left(H_{f}<1\right) \mathcal{H}_{P}$ (more precisely, one considers a particular Banach space of generalized Wick kernels that parametrizes such operators, but for simplicity, we do not make this distinction here), and makes a careful choice of a small polydisc $\mathcal{P} \subset \mathcal{W}$. Furthermore, one introduces a renormalization map $\mathcal{R}: \mathcal{P} \rightarrow \mathcal{P}$, constructed by way of the isospectral smooth Feshbach map, and a rescaling transformation. The idea then is to focus on the dynamical system $(\mathcal{P}, \mathcal{R})$. A key property of $\mathcal{R}$ is that it is contractive on a subspace of $\mathcal{P}$ of finite codimension. Using the smooth Feshbach map, one associates $T_{P}(\rho)$ to an element $H^{(0)} \in \mathcal{P}$, and considers the orbit $\left(H^{(n)}\right)_{n \in \mathbb{N}_{0}}$ under $\mathcal{R}$ that emanates from this initial condition. The elements $H^{(n)}$ of this orbit are called effective Hamiltonians, where $n$ is the scale, and in particular, they are mutually isospectral in the sense of the Feshbach theorem, [BCFS1]. The fixed point of $\mathcal{R}$ on this orbit corresponds to the effective Hamiltonian in the scaling limit, $H^{(\infty)}$, and by isospectrality of the smooth Feshbach map, its spectral properties are directly related to those of $T_{P}(\rho)$.

The main ingredients in this construction are $^{3}$, in addition to the arguments developed in [C], parity invariance, and irrelevance of the $B$-field operator in renormalization group terminology. Indeed, under parity inversion, $x \rightarrow-x$, we have

$$
P \rightarrow-P \quad, \quad P_{f} \rightarrow-P_{f} \quad, \quad A(\rho) \rightarrow-A(\rho),
$$

with respect to which $T_{P}(\rho)$ is evidently invariant. The most general form of the effective Hamiltonian in the scaling limit is

$$
\begin{aligned}
H^{(\infty)}= & \alpha^{(\infty)}(P, \rho) H_{f}+\beta^{(\infty)}(P, \rho) P \cdot P_{f} \\
& +\mu^{(\infty)}(P, \rho) \sigma \cdot P_{f}+\nu^{(\infty)}(P, \rho) \sigma \cdot P,
\end{aligned}
$$

\footnotetext{
${ }^{3}$ T.C. thanks J. Fröhlich for pointing out this key fact.
} 
where the coefficients $\alpha^{(\infty)}(P, \rho), \beta^{(\infty)}(P, \rho), \mu^{(\infty)}(P, \rho)$, and $\nu^{(\infty)}(P, \rho)$ transform trivially under spatial rotations and reflections, and are uniformly bounded in $\rho \geq 0$. Uniform boundedness with respect to $\rho \geq 0$ is in part due to the irrelevance of the $B$-field operator, and absence of interactions is due to the infrared regularization.

The renormalization map $\mathcal{R}$ is constructed in a manner that it commutes with parity inversion, thus all $H^{(n)}, n \in \mathbb{N}_{0}$, and $H^{(\infty)}$ are parity invariant. However, under parity reversal, $\sigma \cdot P$ and $\sigma \cdot P_{f}$ change their signs. Therefore, $\nu(P, \rho)=\mu(P, \rho)=0$, which implies that the ground state energy of $H^{(\infty)}(P, \rho)$, of value 0 , is doubly degenerate. Owing to the isospectrality properties of the smooth Feshbach map, the same statement applies to $T_{P}(\rho)$. This proves (iii).

For the proof of (i) and (iv), we remark that combining parity invariance with the generalized Ward-Takahashi identities of [C], it can be shown that the $\sigma^{0}$-component of the interaction in $H^{(n)}$ is marginal, where $n \in \mathbb{N}_{0}$, while the $\sigma^{i}$-components, for $i=1,2,3$, are irrelevant. Hence, the study of marginal operators in [C] can be straightforwardly adapted to the current problem, and the corresponding results are valid even for $g=1$. This immediately implies (i) and (iv).

To prove (ii), we note that (iv) implies

$$
\left|\partial_{P} E(P, \rho)\right| \geq\left(1-C \alpha^{\delta}\right)|P|
$$

which is bounded away from 0 for all $0 \leq|P| \leq P_{c}$, for $C$ independent of $\rho \geq 0$. Thus, $E(P, \rho)$ has no minima away from 0 for $0 \leq|P| \leq P_{c}$. Furthermore, writing $H_{0}:=H_{f}+\left(P-P_{f}\right)^{2}$ and $T_{P}=H_{0}+W$, we consider

$$
T_{P}=H_{0}+\left(H_{0}+\alpha\right)^{\frac{1}{2}}\left(\left(H_{0}+\alpha\right)^{-\frac{1}{2}} W\left(H_{0}+\alpha\right)^{-\frac{1}{2}}\right)\left(H_{0}+\alpha\right)^{\frac{1}{2}} .
$$

From [C, GLL follows that $\left\|\left(H_{0}+\alpha\right)^{-\frac{1}{2}} W\left(H_{0}+\alpha\right)^{-\frac{1}{2}}\right\| \leq C \sqrt{\alpha}$, hence $T_{P} \geq C\left(H_{0}-\alpha\right)$ for $C \geq \frac{1}{2}$, and $\alpha$ sufficiently small. Since inf $\operatorname{spec}\left\{H_{0}\right\} \geq C_{1} P_{c}^{2}$ for $|P| \geq P_{c}$ with some $C_{1}>0$, it is evident that for all $\rho \geq 0,|E(P, \rho)|$ has its global minimum at $P=0$, such that (ii) follows.

Acknowledgments: S. Vugalter would like to thank H. Spohn for attracting his attention to the results of $[\mathrm{F}]$. T. Chen is deeply indebted to J. Fröhlich for his advice and important comments. He also thanks H. Spohn for discussions. This work was partially supported by the European project HPRN-CT-2002-0027. 


\section{REFERENCES}

[BFS] V. Bach, J. Fröhlich, I. -M.. Sigal, Spectral Analysis for Systems of Atoms and Molecules Coupled to the Quantized Radiation Field, Commun. Math. Phys. 207, no.2, 249-290 (1999)

[BCFS1] V. Bach, T. Chen, J. Fröhlich, I.M. Sigal, Smooth Feshbach Map and operator-theoretic renormalization group methods, to appear in J. Funct. Anal, preprint mp-arc/02-214.

[BCFS2] V. Bach, T. Chen, J. Fröhlich, I.M. Sigal, Determination of the renormalized mass for the Nelson model, in preparation.

$[\mathrm{CH}]$ I. Catto, Ch. Hainzl, Self-energy of one electron in non-relativistic QED, preprint arXiv:math-ph/0207036

[C] T. Chen, Operator-theoretic infrared renormalization and construction of dressed 1-particle states in non-relativistic QED, PhD thesis, ETH Zürich (2001), preprint arXiv:math-ph/0108021

This preprint contains the results for the spinless case $g=0$. Their generalization to the case $g=1$ is in preparation.

[F] J. Fröhlich, Existence of dressed one-electron states in a class of persistent models, Fortschritte der Physik 22, 159-198 (1974)

[GLL] M. Griesemer, E.H. Lieb, M. Loss, Ground states in non-relativistic quantum electrodynamics, Inventiones Math. 145, no.3, 557-595 (2001)

[HVV] Ch. Hainzl, V. Vougalter, S.A. Vugalter, Enhanced binding in nonrelativistic QED, mp.arc 01-455

[H] Ch. Hainzl, One non-relativistic particle coupled to a photon field, preprint: arXiv: math-ph/0202001

[Hi] F. Hiroshima, Self-adjointness of the Pauli-Fierz Hamiltonian for arbitrary values of coupling constants, Ann. Henri Poincaré 3, 171-201 (2002)

[HS1] F. Hiroshima, H. Spohn, Enhanced Binding through coupling to a Quantum Field, preprint mp.arc 01-39

[HS2] F. Hiroshima, H. Spohn, Ground state degeneracy of the Pauli-Fierz Hamiltonian with spin, preprint math-ph/0205018

[VZ] S.A. Vugalter, G.M. Zhislin, The Symmetry and Efimov's Effect in Systems of Three-Quantum Particles, Commun. Math. Phys. 87, no. 1, 89-103 $(1982 / 1983)$

${ }^{1}$ Courant Institute of Mathematical Sciences, New York UniverSity, 251 Mercer Street, NY 10012-1185, USA

E-mail address: chenthom@cims.nyu.edu

1 Department of Mathematics and Statistics, McMaster UniverSity, 1280 Main Street West , Hamilton, Ontario L8S 4K1, Canada

E-mail address: vougav@math.mcmaster.ca

2 Mathematisches Institut, LMU München, Theresienstrasse 39, 80333 Munich, Germany

E-mail address: wugalter@mathematik.uni-muenchen.de 